Homology, Homotopy and Applications, vol.15(1), 2013, pp.27-49

\title{
ÉTALE HOMOTOPY TYPES AND BISIMPLICIAL HYPERCOVERS
}

\author{
MICHAEL D. MISAMORE \\ (communicated by Gunnar Carlsson)
}

\begin{abstract}
Suppose $(\mathcal{C}, x)$ is a pointed locally connected small Grothendieck site, and let $(X, z)$ denote any connected locally fibrant simplicial sheaf $X$ equipped with a "geometric" point $z$. Following Artin-Mazur, an étale homotopy type of $X$ may then be defined via the geometrically pointed hypercovers of $X$ to yield a pro-object of the homotopy category, but this is not the only possible definition. In Étale homotopy of simplicial schemes, Friedlander defined another étale homotopy type of a simplicial scheme $X$ by taking diagonals of geometrically pointed bisimplicial hypercovers. In this paper, these two types are shown to be pro-isomorphic by means of a direct comparison of the associated cocycle categories. Friedlander's construction of étale homotopy types as actual pro-simplicial sets relies on a rigidity property of the étale topology that may not always be available for arbitrary sites; the cocycle methods employed here do not have this limitation. By consequence, the associated homotopy types constructed from hypercovers and bisimplicial hypercovers are shown to be pro-isomorphic on any locally connected small Grothendieck site, and the comparison at the level of cocycles shows, in particular, that both abelian and nonabelian sheaf cohomology may be computed via bisimplicial hypercovers on arbitrary small Grothendieck sites.
\end{abstract}

\section{Introduction}

In classical étale homotopy theory, the étale homotopy type of a geometrically pointed connected locally noetherian scheme $(X, z)$ is defined by taking objectwise connected components $\Pi(U, u)$ of the system of all pointed hypercovers $(U, u) \rightarrow X$ of $X$ and pointed simplicial homotopy classes of maps between them. This system is cofiltered and thus results in a pro-object of $\mathrm{Ho}\left(\mathbf{s S e t}_{*}\right)$, the homotopy category of pointed path-connected simplicial sets. This is the classical étale homotopy type of Artin-Mazur [1]. On the other hand, if one starts with a geometrically pointed connected locally noetherian simplicial scheme $X$, then one has to make a choice about

Received August 27, 2010, revised December 26, 2011; published on March 4, 2013.

2000 Mathematics Subject Classification: 18G30, 14F35.

Key words and phrases: étale, homotopy, simplicial, sheaf.

Article available at http://intlpress.com/HHA/v15/n1/a2 and doi:10.4310/HHA.2013.v15.n1.a2

Copyright (C) 2013, International Press. Permission to copy for private use granted. 
what types of hypercovers of $X$ to consider. The choice taken by Friedlander was to define his étale topological type via the system of (rigid) pointed bisimplicial hypercovers $(U, u) \rightarrow X$ of $X$ and fibrewise (over $X$ ) pointed simplicial homotopy classes of maps between them, where a bisimplicial hypercover $U \rightarrow X$ is a map of bisimplicial schemes such that each degreewise map $U_{n} \rightarrow X_{n}$ is a hypercover of the scheme $X_{n}$ for $n \geqslant 0$. There one must take diagonals $d(U, u)$ followed by connected components in order to produce a pro-object of $\mathrm{Ho}\left(\mathbf{s S e t}_{*}\right)$, and it is obvious that this specializes to the Artin-Mazur definition for geometrically pointed schemes $X$ regarded as constant simplicial schemes. In [5], Isaksen introduced another model for this homotopy type by taking the "realization" of the diagram of (rigid) étale homotopy types Ét $\left(X_{n}\right)$ of the constituent schemes and showed, in particular, that his model (also taking values in pro-simplicial sets) is weakly equivalent to Friedlander's. Regardless of the model in pro-simplicial sets, the resulting homotopy type of interest may be taken to be the pro-object $T_{b}(X, z)$ of Ho(sSet $\left.{ }_{*}\right)$ defined by means of connected components of diagonals of geometrically pointed bisimplicial hypercovers of $X$.

There is another possible choice for a system of geometrically pointed hypercovers of a geometrically pointed simplicial scheme: one knows (for various reasons) that a pointed hypercover $(U, u) \rightarrow(X, z)$ should be defined as a pointed local trivial fibration on the relevant site, so one may simply take these instead of the bisimplicial hypercovers. These were apparently first considered for the purpose of defining a homotopy type by Schmidt in [12]. From this point of view it is natural to drop the requirement that $X$ be representable by a simplicial scheme and instead consider the system of geometrically pointed hypercovers of $X$ and pointed simplicial homotopy classes of maps between them for any geometrically pointed connected locally fibrant simplicial sheaf $(X, z)$, where the words "geometrically pointed" are suitably defined. One way or another, it has been known for some time that this also results in a pro-object $T(X, z)$ of $\mathrm{Ho}\left(\mathbf{s S e t}_{*}\right)$; the underlying ideas go back to Brown's thesis [2].

Unfortunately, the rigidity techniques of $[\mathbf{3}]$ do not automatically extend to arbitrary small Grothendieck sites; in particular, there are Grothendieck toposes that do not possess any points at all, and one does not know a priori that the required rigidity property of covers is automatically satisfied when one is not using the usual étale topology. However, the homotopy types $T(X, z)$ and $T_{b}(X, z)$ (and unpointed analogues) still exist, and one may ask how to compare them.

Here this comparison is achieved by working at the level of cocycle categories in the sense of $[\mathbf{1 0}]$ : it is shown here (Theorem 4.18) that there are bijections

$$
\pi_{0} H_{\text {hyp }}(x, y) \cong \pi_{0} H_{\text {bihyp }}(x, y) \cong[x, y]
$$

between the path components of the cocycle categories for ordinary and bisimplicial hypercovers for any locally fibrant pointed simplicial sheaf $y$ on the ambient small Grothendieck site $\mathcal{C}$. For these bijections one only requires that the site $\mathcal{C}$ be pointed (and only if one desires to speak about pointed hypercovers). They do not depend on "having enough points" or rigidity properties of the ambient small Grothendieck site, and therefore constitute the correct general argument.

As this is a foundational result about a comparison of pro-homotopy types, its applications are limited to the transfer of facts about the first type to the second. For 
the sake of example, the identifications

$$
H^{n}(X, H) \cong \lim _{p: d \overrightarrow{d(U)} \rightarrow X} H^{n}(d U, H)
$$

for sheaves of groups $H$ are established in Proposition 5.2. These results are proven without spectral sequence arguments and work equally well for nonabelian $H^{1}([\mathbf{3}, 3.8]$ only addressed the abelian case). This identification is usually given for hypercovers using the ordinary Verdier hypercovering theorem; the fact that it also works for bisimplicial hypercovers is an immediate application of the comparison given above, and again it is true for arbitrary small Grothendieck sites, not just étale sites.

Finally, it is shown here in Theorem 5.3 that $T_{b}(X, z)$ is indeed pro-isomorphic to $T(X, z)$ whenever $(X, z)$ is a pointed connected locally fibrant simplicial sheaf on a pointed locally connected site where the distinguished "point" is determined by some object $\Omega$ representing a sheaf (such as a geometric point). The resulting invariance of $T_{b}(X, z)$ up to pro-isomorphism under (pointed) local weak equivalences is the subject of Corollary 5.5. The $\mathrm{Ex}^{\infty}$ functor is employed in Lemma 5.4 to demonstrate that this invariance holds without any fibrancy assumptions on $(X, z)$. One recovers, in particular, the fact that a bisimplicial hypercover $U \rightarrow X$ determines a pro-isomorphism $T_{b}(d U) \cong T_{b}(X)$ of bisimplicial étale homotopy types (cf. $[\mathbf{3}, 8.1]$ ); the proof here is elementary and does not make use of the pro-Whitehead theorem from $[\mathbf{1}, \S 4]$.

\section{Hypercovers and bisimplicial hypercovers}

A map $U \rightarrow X$ of simplicial (pre)sheaves on a small Grothendieck site $\mathcal{C}$ is called a hypercover if it is a local fibration and a local weak equivalence (cf. [6] for a definition and discussion of the local right lifting property defining local fibrations), and it is well known that when $X=K(X, 0)$ is the discrete or "constant" simplicial (pre)sheaf associated to an object $X$ of $\mathcal{C}$, the map $U \rightarrow X$ as above is a hypercover in this sense exactly when the maps

$$
\begin{aligned}
U_{0} & \rightarrow X_{0} \\
U_{n} & \rightarrow \operatorname{cosk}_{n-1} U_{n}
\end{aligned}
$$

are local epimorphisms of (pre)sheaves on $\mathcal{C}$ for $n \geqslant 1$, which may be taken as the "classical" definition. The fact that these definitions correspond follows from ([7, 1.12]) in the case where $X$ is locally fibrant, or by a Boolean localization argument in the general case, following Jardine $[\mathbf{9}]$.

Suppose $\mathcal{C}$ is pointed in the sense that there is a geometric morphism

$$
x: \operatorname{Set} \rightarrow \operatorname{Shv}(\mathcal{C})
$$

of toposes. For the purposes of this paper, a pointed simplicial sheaf $(X, z)$ on $\mathcal{C}$ will be a simplicial sheaf $X$ on $\mathcal{C}$ together with a choice of section $z \in x^{*}\left(X_{0}\right)$, and a pointed map $f:(X, z) \rightarrow\left(Y, z^{\prime}\right)$ of pointed simplicial sheaves will be a map $X \rightarrow Y$ of the underlying simplicial sheaves such that $x^{*}(f)(z)=z^{\prime}$. In the usual geometric setting for étale homotopy theory, such "points" $z$ correspond to geometric points of $X$ whenever $X=K(X, 0)$ is a discrete representable simplicial sheaf. A pointed hypercover $(U, u) \rightarrow(X, z)$ of a pointed simplicial sheaf $(X, z)$ on a pointed small 
Grothendieck site $(\mathcal{C}, x)$ will be a hypercover $U \rightarrow X$ that is a pointed map (with respect to $x$ ) of simplicial sheaves, and a pointed map of pointed hypercovers of $(X, z)$ will be a pointed map over $X$ of the underlying simplicial sheaves. It has been observed as early as $[\mathbf{2}]$ that the pointed hypercovers of any locally fibrant pointed simplicial sheaf $(X, z)$ together with pointed simplicial homotopy classes of maps between them over $(X, z)$ form a cofiltered category, here denoted $\operatorname{HR}_{*}(X, z)$.

A bisimplicial hypercover $f: U \rightarrow X$ of a simplicial (pre)sheaf $X$ on a small Grothendieck site $\mathcal{C}$ is a map of bisimplicial (pre)sheaves $f: U \rightarrow K_{v}(X, 0)$, where $X$ is being regarded as simplicially discrete in the "vertical" direction, such that each of the constituent maps $f_{m}: U_{m} \rightarrow X_{m}$ in "horizontal" degree $m \geqslant 0$ is a hypercover. If $(\mathcal{C}, x)$ is a pointed site, then a pointed bisimplicial hypercover $f:(U, u) \rightarrow(X, z)$ of a pointed simplicial sheaf $(X, z)$ is a bisimplicial hypercover $f: U \rightarrow X$ in sheaves such that $x^{*}(f)(u)=z$, where $u \in x^{*}\left(U_{0,0}\right)$ is the "point" associated to $U$. A pointed map $f:(V, v) \rightarrow(U, u)$ of pointed bisimplicial hypercovers of a pointed simplicial sheaf $(X, z)$ is a map of bisimplicial hypercovers $f: V \rightarrow U$ over $X$ such that $x^{*}(f)(v)=u$. The most significant fact about these objects for the present purposes is given by

Proposition 2.1. If $f:(U, u) \rightarrow(X, z)$ is a bisimplicial hypercover of a pointed simplicial sheaf $(X, z)$ on a pointed small Grothendieck site $(\mathcal{C}, x)$, then the map $(d U, u) \rightarrow$ $(X, z)$ of simplicial sheaves induced by taking diagonals is a local weak equivalence.

Proof. Fixing a Boolean localization $p: \operatorname{Shv}(\mathcal{B}) \rightarrow \operatorname{Shv}(\mathcal{C})$, it suffices to show that the induced map $p^{*}(d U) \rightarrow p^{*}(X)$ is a sectionwise weak equivalence by techniques of $[\mathbf{9}]$, but this follows from the corresponding fact for simplicial sets $([4,1.7, \mathrm{IV}])$. The pointedness of the induced map is trivial.

This proposition and $([\mathbf{3}, 8.1])$ serve as motivation for asking whether any local weak equivalence induces isomorphisms on the étale homotopy pro-groups of the associated bisimplicial étale homotopy types. Reader beware: the diagonal of a pointwise fibration of simplicial sets need not be a fibration in general, so one does not generally expect the diagonal of a bisimplicial hypercover to be a hypercover. This is a source of technical problems when comparing the differing definitions of étale homotopy types.

\section{The étale homotopy type of a simplicial sheaf}

Say that a small Grothendieck site $\mathcal{C}$ is locally connected if there exists a functor $\Pi: \operatorname{Shv}(\mathcal{C}) \rightarrow$ Set left adjoint to the constant sheaf functor $\Gamma^{*}: \operatorname{Set} \rightarrow \operatorname{Shv}(\mathcal{C})$, and say that a locally connected site $\mathcal{C}$ is connected if $\Pi(*)=*$, where $*$ denotes the terminal sheaf on $\mathcal{C}$. In geometric situations the functor $\Pi$ is that induced by the functor which sends any scheme to its set of connected scheme-theoretic components. A simplicial sheaf $X$ on a connected site $\mathcal{C}$ will be called connected if $\pi_{0} \Pi(X) \cong *$; a quick argument using $H^{0}\left(-, K\left(\Gamma^{*} S, 0\right)\right)$ for variable sets $S$ shows that $U$ is connected whenever $U \rightarrow X$ is a hypercover of a connected simplicial sheaf $X$, and a similar statement is true for diagonals of bisimplicial hypercovers by the same argument.

Suppose $(\mathcal{C}, x)$ is a pointed locally connected small Grothendieck site and that $(X, z)$ is a pointed (with respect to $x)$ connected locally fibrant simplicial sheaf on $\mathcal{C}$. Then the pointed hypercovers of $(X, z)$ are cofiltered up to simplicial homotopy so one may proceed to define an "étale" homotopy type $T(X, z)$ for $(X, z)$ : it is the 
pro-object in $\mathrm{Ho}\left(\mathbf{s S e t}_{*}\right)$ given by applying $\Pi$ to the cofiltered diagram $\operatorname{HR}_{*}(X, z)$ of pointed hypercovers of $(X, z)$ and pointed simplicial homotopy classes of maps between them. This definition applies, in particular, to pointed locally fibrant connected simplicial schemes $(X, z)$ on étale sites, and is clearly not the same as the étale topological type of Friedlander defined by means of diagonals of (rigid) pointed bisimplicial hypercovers of $(X, z)$. Nevertheless $T(X, z)$ has several good properties: firstly, it specializes to the classical étale homotopy type for geometrically pointed connected schemes (this is a matter of checking definitions). Secondly, the fact that it is defined in terms of not-necessarily-representable hypercovers does not matter:

Proposition 3.1. For any geometrically pointed connected scheme $(X, z)$ on a locally connected étale site, the étale homotopy type $T(X, z)$ defined here is pro-isomorphic to the classical étale homotopy type Ét $(X, z)$ of Artin-Mazur defined by means of pointed representable hypercovers of $(X, z)$.

Proof. The only point is to show that any pointed hypercover $(U, u) \rightarrow(X, z)$ of a scheme $X$ can be refined by a pointed representable hypercover (as then the result follows by a cofinality argument in $\left.\mathrm{HR}_{*}(X, z)\right)$. This construction was given by Jardine in $[\mathbf{8}]$ based on the work of Artin-Mazur $[\mathbf{1}, \S 8])$.

Next, one may show directly that the type $T(U, u)$ is pro-isomorphic to the type $T(X, z)$ for any pointed hypercover $(U, u) \rightarrow(X, z)$ of $(X, z)$. The corresponding (actually weaker) statement for the étale topological type of Friedlander requires some work to establish (cf. $[3,8.1]$ ) but is easy to prove for $T(-,-)$ :

Lemma 3.2. Suppose $(\mathcal{C}, x)$ is a pointed locally connected small Grothendieck site, $(X, z)$ a pointed connected locally fibrant simplicial sheaf on $\mathcal{C}$, and $f:(U, u) \rightarrow(X, z)$ a pointed hypercover of $(X, z)$. Then $f$ induces a pro-isomorphism $T(U, u) \cong T(X, z)$.

Proof. Consider the slice category $\operatorname{HR}_{*}(X, z) / f$ whose objects are the commutative triangles

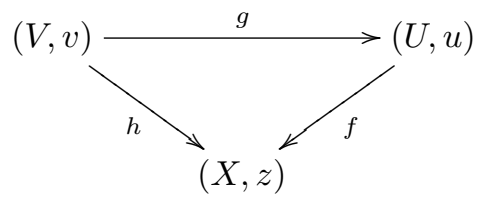

over $(X, z)$, where $h$ is a hypercover. The maps $g$ may not be hypercovers themselves, but as any such $V$ and $U$ are locally fibrant (since $X$ is), any such object has a functorial refinement up to weak equivalence by an object $g^{\prime}:(Z, z) \rightarrow(U, u)$ over $(X, z)$ such that $g^{\prime}$ is a pointed hypercover of $(U, u)$ : this is determined by the usual factorization

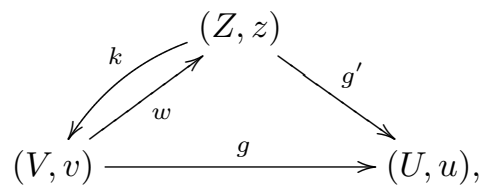

where $g^{\prime}$ is a local fibration (and local weak equivalence by closed model axiom CM2) and $w$ is a local weak equivalence that is a section of the local trivial fibration $k$. This 
determines a full cofinal subcategory

$$
i:\left(\operatorname{HR}_{*}(X) / f\right)_{\text {hyp }} \hookrightarrow \operatorname{HR}_{*}(X) / f
$$

as one can see by equalizing any two maps of pointed hypercovers over $X$ up to pointed simplicial homotopy and replacing the resulting equalizer $(E, e) \rightarrow(U, u)$ over $X$ by a hypercover $\left(E^{\prime}, e^{\prime}\right) \rightarrow(U, u)$ by factorization as above. There is an equivalence $f_{*}: \operatorname{HR}_{*}(U) \simeq\left(\operatorname{HR}_{*}(X) / f\right)_{\text {hyp }}$ defined by composing with $f$ or likewise forgetting the maps to $X$. Further, the functor $p: \operatorname{HR}_{*}(X) / f \rightarrow \operatorname{HR}_{*}(X)$ defined by forgetting the maps to $f$ is cofinal since $\operatorname{HR}_{*}(X)$ is cofiltered. It follows that the composite

$$
\operatorname{HR}_{*}(U) \stackrel{f_{*}}{\longrightarrow}\left(\operatorname{HR}_{*}(X) / f\right)_{\text {hyp }} \stackrel{p i}{\longrightarrow} \operatorname{HR}_{*}(X)
$$

is cofinal, and this composite induces the desired pro-isomorphism.

This cofinality argument works essentially because $T(-,-)$ treats both the base simplicial sheaf $X$ and its pointed hypercovers on the same footing; such an argument therefore fails for bisimplicial hypercovers of simplicial sheaves. It almost immediately follows that $T(X, z)$ is invariant up to pro-isomorphism under pointed local weak equivalences:

Proposition 3.3. Suppose $(\mathcal{C}, x)$ is a pointed locally connected small Grothendieck site, $(X, z)$ and $(Y, y)$ pointed connected locally fibrant simplicial sheaves on $\mathcal{C}$, and $f:(Y, y) \rightarrow(X, z)$ a pointed local weak equivalence. Then $f$ induces a pro-isomorphism $T(Y, y) \cong T(X, z)$.

Proof. As $Y$ and $X$ are locally fibrant there is a factorization

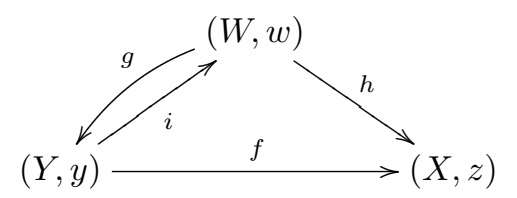

of the map $f$, where $g$ and $h$ are pointed hypercovers and $i$ is right inverse to $g$. The induced map $i_{*}: T(Y, y) \rightarrow T(W, w)$ is right inverse to the induced map $g_{*}$, which is a pro-isomorphism by Lemma 3.2, so $i_{*}$ is also left inverse to $g_{*}$ and a pro-isomorphism. The map $h_{*}$ is also a pro-isomorphism by the lemma so $h_{*} i_{*}=f_{*}: T(Y, y) \rightarrow T(X, z)$ is a pro-isomorphism.

In an earlier work [12], Schmidt observed that this latter fact is implied by an application of the generalized Verdier hypercovering theorem; the present proof is included because it may be of independent interest. To give another comparison with known results, recall that in $([\mathbf{5}, 2])$ Isaksen shows this his étale realization functor $\operatorname{Re}^{e t}$ is left Quillen for the local projective structure so that it sends local weak equivalences between local projective cofibrant simplicial presheaves to weak equivalences of prosimplicial sets. The type $T(-,-)$ defined here sends pointed local weak equivalences between pointed connected locally fibrant simplicial sheaves to pro-isomorphisms in Ho(sSet $\left.{ }_{*}\right)$. Obviously the points are only there to make a comparison of homotopy pro-groups; this general line of argument continues to work in the unpointed case. 


\section{Cocycles for bisimplicial sheaves}

In what follows $\mathcal{C}$ will be an arbitrary small Grothendieck site and $\mathbf{S e t} \rightarrow \mathbf{S h v}(\mathcal{C})$ a point of $\mathcal{C}$ (or no point at all in the unpointed situation). Familiarity with the definitions of $[\mathbf{1 0}]$ and $[\mathbf{1 1}]$ will be assumed. The word "pointed" will always mean with respect to the chosen point of $\mathcal{C}$ rather than with respect to the terminal sheaf. Pointed (bi)simplicial sheaves on $\mathcal{C}$ will be denoted from now on simply with lowercase letters to reduce the notational burden, and unless otherwise specified any map $x \rightarrow y$ between pointed (bi)simplicial sheaves will be pointed. The underlying site will always be $\mathcal{C}$.

For any two pointed simplicial sheaves $x, z$ on $\mathcal{C}$, there is a category $H_{\text {bihyp }}(x, z)$ of cocycles of the form

$$
x \stackrel{d(f) \simeq}{\longleftarrow} d(u) \stackrel{p}{\rightarrow} z,
$$

where $f: u \rightarrow x$ is a pointed bisimplicial hypercover of $x$ and $d$ is the diagonal functor, whose morphisms are commutative diagrams

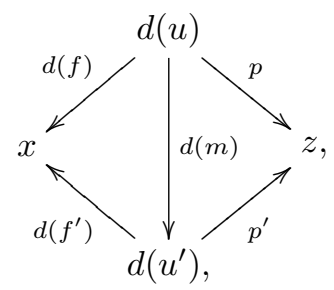

where $m: u \rightarrow u^{\prime}$ is any pointed map of bisimplicial hypercovers of $x$.

As these categories turn out to be a bit tricky to study directly, one may also consider categories $H_{d}(x, z)$ whose objects are cocycles of the form

$$
x \stackrel{d(f) \simeq}{\longleftarrow} d(u) \stackrel{p}{\rightarrow} z,
$$

where $f: u \rightarrow x$ is any pointed map of bisimplicial sheaves that is a diagonal local weak equivalence in the sense that $d(f)$ is a local weak equivalence of simplicial sheaves, and whose morphisms are similarly defined; it is immediate from the definition that $H_{\text {bihyp }}(x, z)$ is a full subcategory of $H_{d}(x, z)$ for any fixed $x$ and $z$.

Recall the Moerdijk closed model structure for bisimplicial sets: the fibrations (resp. weak equivalences) are, by definition, the diagonal fibrations (resp. diagonal weak equivalences), and the cofibrations are defined by the left lifting property with respect to all trivial fibrations (cf. $[\mathbf{4}, 3.15$, IV]). Every Moerdijk cofibration is a monomorphism of bisimplicial sets and therefore a diagonal cofibration in particular.

The diagonal functor $d$ has a right adjoint $d_{*}([4,3.13, \mathrm{IV}])$ so that any object

$$
x \stackrel{d(f) \simeq}{\longleftarrow} d(u) \stackrel{p}{\rightarrow} z
$$

of $H_{d}(x, z)$ is uniquely identified with a diagram

$$
x \stackrel{f}{\leftarrow} u \stackrel{\tilde{p}}{\rightarrow} d_{*}(z)
$$

where $f$ is the underlying map of bisimplicial sheaves and $\tilde{p}$ is the adjoint of $p$. In other words any such cocycle $(d(f), p)$ may be identified with a "cocycle" $(f, \tilde{p})$. 
Lemma 4.1. If $f: X \rightarrow Y$ is a trivial fibration of simplicial sets, then the induced map $d_{*}(f): d_{*} X \rightarrow d_{*} Y$ is a trivial fibration for the Moerdijk closed model structure on bisimplicial sets.

Proof. It must be shown that $d_{*}(f)$ has the right lifting property with respect to any Moerdijk cofibration $i: A \rightarrow B$. By adjointness, this is equivalent to showing that $f$ has the right lifting property with respect to $d(i)$, but $d(i)$ is a cofibration of simplicial sets since $i: A \rightarrow B$ is, in particular, a monomorphism of bisimplicial sets $([4,3.15$, IV]). The required lift exists since $f$ is a trivial fibration.

Corollary 4.2. If $f: X \rightarrow Y$ is a weak equivalence of fibrant simplicial sets, then the induced $\operatorname{map}_{*}(f): d_{*} X \rightarrow d_{*} Y$ is a diagonal weak equivalence of bisimplicial sets.

Proof. Factor the map $f$ as a weak equivalence $\sigma$ followed by trivial fibration $g$ such that $\sigma$ is a section of a trivial fibration $h$. Then $d_{*}(h)$ and $d_{*}(g)$ are trivial fibrations for the Moerdijk structure by Lemma 4.1, so $d_{*}(f)$ is a diagonal equivalence.

The "localized" version of this is then given by

Lemma 4.3. If $\beta: z \rightarrow z^{\prime}$ is a local weak equivalence of pointed locally fibrant simplicial sheaves, then $d_{*}(\beta)$ is a pointed diagonal local weak equivalence.

Proof. Fix a Boolean localization $p: \operatorname{Shv}(\mathcal{B}) \rightarrow \operatorname{Shv}(\mathcal{C})$. Then the map $p^{*}(\beta)$ is a sectionwise weak equivalence of sectionwise fibrant simplicial sheaves on $\mathcal{B}$ so that $d_{*}\left(p^{*}(\beta)\right)$ is a sectionwise diagonal weak equivalence of bisimplicial sheaves by Lemma 4.2. In bisimplicial degree $(m, n)$ this map is given by the sheaf map $\left(p^{*} \beta\right)^{\Delta^{m} \times \Delta^{n}}$ where $\Delta^{m} \times \Delta^{n}$ is the constant sheaf associated to the corresponding simplicial set. As $p^{*}$ is exact one has $p^{*}\left(\Delta^{m} \times \Delta^{n}\right)=\Delta^{m} \times \Delta^{n}$ so that the map $\left(p^{*} \beta\right)^{\Delta^{m} \times \Delta^{n}}$ is isomorphic to the map $p^{*}\left(\beta^{\Delta^{m} \times \Delta^{n}}\right)$ naturally in $m, n \geqslant 0$. Thus the map $p^{*}\left(d_{*}(\beta)\right)$ is also a sectionwise diagonal weak equivalence. By exactness of $d$ and $p^{*}$, it follows that $p^{*}\left(d\left(d_{*}(\beta)\right)\right)$ is a sectionwise weak equivalence so that $d\left(d_{*}(\beta)\right)$ must be a local weak equivalence; hence $d_{*}(\beta)$ is a diagonal local weak equivalence as was to be shown.

Here is a first application to cocycles:

Lemma 4.4. If $\beta: z \rightarrow z^{\prime}$ is a local weak equivalence of pointed locally fibrant simplicial sheaves, then the induced functor

$$
\beta_{*}: B H_{d}(x, z) \rightarrow B H_{d}\left(x, z^{\prime}\right)
$$

is a homotopy equivalence.

Proof. The functor $\beta_{*}$ is defined by composition with $\beta$, sending the right-hand map from $p$ to $p^{\prime}:=\beta p$. The local weak equivalence $\beta$ determines a diagonal local weak equivalence $d_{*}(\beta)$ by Lemma 4.3 , and by adjunction the associated "cocycle" $(f, \tilde{p})$ is sent to $\tilde{p}^{\prime}:=d_{*}(\beta) \tilde{p}$.

Suppose one has a pointed "cocycle" of the form

$$
x \stackrel{f}{\leftarrow} u \stackrel{\tilde{p}}{\rightarrow} d_{*}\left(z^{\prime}\right),
$$

where $f$ is a diagonal local weak equivalence. Emulating the proof of Lemma 1 of [10], this is equivalent to giving a map $(f, \tilde{p}): u \rightarrow x \times d_{*}\left(z^{\prime}\right)$ which may be factored using 
the Moerdijk structure in sections as

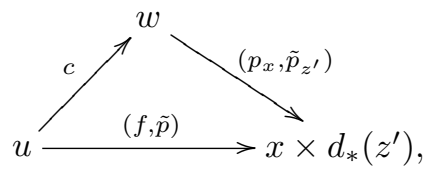

where $c$ is a sectionwise diagonal equivalence and $\left(p_{x}, \tilde{p}_{z^{\prime}}\right)$ is a sectionwise diagonal fibration. Observe that $p_{x}$ is a diagonal local weak equivalence since both $f$ and $c$ are. Pull back along the diagonal equivalence $1 \times d_{*}(\beta)$ to get a commutative square

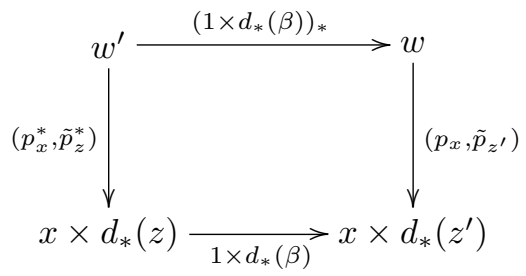

defining $w^{\prime}$. The map $\left(p_{x}^{*}, \tilde{p}_{z}^{*}\right)$ is a sectionwise diagonal fibration since $\left(p_{x}, \tilde{p}_{z^{\prime}}\right)$ was, and passing to a Boolean localization $p^{*}$ preserves pullbacks (and $d$ is exact) so the map $\left(1 \times d_{*}(\beta)\right)_{*}$ is a diagonal local weak equivalence and therefore so is the map $p_{x}^{*}$. This determines a functor $\tilde{\psi}$ from the category of pointed "cocycles" of the form

$$
x \stackrel{f}{\leftarrow} u \stackrel{\tilde{p}}{\rightarrow} d_{*}\left(z^{\prime}\right)
$$

to the analogous category of such objects with target $d_{*}(z)$. The canonical maps

$$
(f, \tilde{p}) \rightarrow\left(p_{x}, \tilde{p}_{z^{\prime}}\right) \leftarrow \beta_{*} \tilde{\psi}(f, \tilde{p})
$$

and

$$
(g, \tilde{q}) \rightarrow \tilde{\psi} \beta_{*}(g, \tilde{q})
$$

determine natural transformations (use that $w^{\prime}$ is a pullback for the latter). The aforementioned categories are therefore homotopy equivalent. These categories are isomorphic to $H_{d}(x, z)$ and $H_{d}\left(x, z^{\prime}\right)$, respectively, so the result follows.

Corollary 4.5. If $\beta: y \rightarrow z$ is a globally fibrant model of a pointed locally fibrant simplicial sheaf $y$, then the induced map

$$
\pi_{0}\left(\beta_{*}\right): \pi_{0} H_{d}(x, y) \rightarrow \pi_{0} H_{d}(x, z)
$$

is a bijection.

Proof. Globally fibrant objects are locally fibrant. Apply Lemma 4.4.

In Lemma 4 of [11], Jardine established that if

$$
x \stackrel{f \simeq}{\longleftarrow} u \rightarrow z
$$

is any cocycle of pointed simplicial (pre)sheaves with $z$ locally fibrant, then it may be functorially replaced by a cocycle of the form

$$
x \stackrel{f^{\prime} \simeq}{\longleftarrow} u^{\prime} \rightarrow z,
$$

where $f^{\prime}$ is a hypercover such that the new cocycle is in the same path component of 
$H(x, z)$ as the original. It follows that the inclusion functor

$$
j: H_{\text {hyp }}(x, z) \hookrightarrow H(x, z)
$$

of the full subcategory $H_{\text {hyp }}(x, z)$ into $H(x, z)$ induces a bijection on path components for any locally fibrant simplicial (pre)sheaf $z$.

To give an analogue for $H_{d}(x, z)$, let $H_{d-\text { hyp }}(x, z)$ denote the category of cocycles of the form

$$
x \stackrel{d(f) \simeq}{\longleftarrow} d(u) \rightarrow z
$$

where $f: u \rightarrow x$ is any map of pointed bisimplicial sheaves to a pointed simplicial sheaf $x$ such that the induced map $d(f)$ is a hypercover, whose morphisms are given by maps $m: u \rightarrow u^{\prime}$ inducing maps of such cocycles in the usual way. Then $H_{d-\text { hyp }}(x, z)$ is another full subcategory of $H_{d}(x, z)$ so that the inclusion functor

$$
i^{\prime}: H_{d-\text { hyp }}(x, z) \hookrightarrow H_{d}(x, z)
$$

is injective on path components.

Say that a map $f: x \rightarrow y$ of pointed bisimplicial sheaves is a diagonal local fibration if the induced map $d(f)$ is a local fibration of simplicial sheaves.

Lemma 4.6. If $z$ is a pointed locally fibrant simplicial sheaf on $\mathcal{C}$, then $d_{*}(z) \rightarrow *$ is a diagonal local fibration.

Proof. Fix a Boolean localization $p: \operatorname{Shv}(\mathcal{B}) \rightarrow \operatorname{Shv}(\mathcal{C})$. The map $p^{*}(z) \rightarrow *$ is a sectionwise fibration since $z$ is locally fibrant, so the induced map $d_{*}\left(p^{*}(z)\right) \rightarrow *$ is a sectionwise diagonal fibration by $([4,3.14, \mathrm{IV}])$. By the proof of Lemma 4.3 this implies that the map $p^{*}\left(d_{*}(z)\right) \rightarrow *$ is also a sectionwise diagonal fibration, but by exactness this implies that $p^{*}\left(d\left(d_{*}(z)\right)\right) \rightarrow *$ is a sectionwise fibration so that $d\left(d_{*}(z)\right) \rightarrow *$ is a local fibration; thus $d_{*}(z) \rightarrow *$ is a diagonal local fibration.

Lemma 4.7. If $z$ is locally fibrant, then the induced map $\pi_{0}\left(i^{\prime}\right)$ is a bijection.

Proof. As above, identify any object

$$
x \stackrel{d(f)}{\longleftarrow} u \stackrel{p}{\rightarrow} z
$$

of $H_{d}(x, z)$ with the corresponding map

$$
(f, \tilde{p}): u \rightarrow x \times d_{*}(z)
$$

and factor $(f, \tilde{p})$ as a sectionwise trivial Moerdijk cofibration $c: u \rightarrow w$ followed by a sectionwise Moerdijk fibration $\left(p_{x}, \tilde{p}_{z}\right): w \rightarrow x \times d_{*}(z)$. Then $c$ and $f$ are diagonal local weak equivalences so $p_{x}$ is a diagonal local weak equivalence. The map $p_{x}$ is the composite

$$
w \stackrel{\left(p_{x}, p_{z}\right)}{\longrightarrow} x \times d_{*}(z) \stackrel{\mathrm{pr}_{x}}{\longrightarrow} x
$$

where the first map is a sectionwise diagonal fibration and the second map is a diagonal local fibration since $d_{*}(z) \rightarrow *$ is a diagonal local fibration. Thus $d\left(p_{x}\right)$ is a hypercover, and the result follows by adjunction. 
The category $H_{d-\text { hyp }}(x, z)$ is a (not a priori full) subcategory of $H_{\text {hyp }}(x, z)$, the full subcategory of $H(x, z)$ whose objects are cocycles of the form

$$
x \stackrel{f \simeq}{\longleftarrow} v \rightarrow z,
$$

where $f$ is any hypercover. Let

$$
i^{\prime \prime}: H_{d-\mathrm{hyp}}(x, z) \hookrightarrow H_{\mathrm{hyp}}(x, z)
$$

denote the inclusion.

Lemma 4.8. For any pointed locally fibrant simplicial sheaf $z$ the induced map $\pi_{0}\left(i^{\prime \prime}\right)$ is surjective.

Proof. Let

$$
x \stackrel{f \simeq}{\longleftarrow} v \stackrel{p}{\rightarrow} z
$$

be any object of $H_{\text {hyp }}(x, z)$ and identify it with the map $(f, p): v \rightarrow x \times z$. Factor $(f, p)$ as a sectionwise trivial Moerdijk cofibration $c: v \rightarrow w$ followed by a sectionwise Moerdijk fibration $\left(p_{x}, p_{z}\right): w \rightarrow x \times z$. The maps $f$ and $c$ are diagonal local weak equivalences so $p_{x}$ is a diagonal local weak equivalence. The map $p_{x}$ is the composite

$$
w \stackrel{\left(p_{x}, p_{z}\right)}{\longrightarrow} x \times z \stackrel{\mathrm{pr}_{x}}{\longrightarrow} x,
$$

where the first map is a sectionwise diagonal fibration and the second map is a diagonal local fibration since $z$ is locally fibrant. Thus $d\left(p_{x}\right)$ is a hypercover, so the result follows.

Recall that the diagonal functor $d$ also has a left adjoint $d^{*}(3.3, \mathrm{IV},[4])$. To show injectivity of $\pi_{0}\left(i^{\prime \prime}\right)$ one may use a roundabout argument beginning with

Lemma 4.9. For any simplicial set $X$, the unit map $\eta: X \rightarrow d d^{*}(X)$ is a weak equivalence.

Proof. Any Moerdijk cofibration $c$ is a monomorphism $[4,3.15$, IV], so $d(c)$ is a monomorphism and hence a cofibration of simplicial sets. If $e$ is any Moerdijk weak equivalence, then $d(e)$ is a weak equivalence of simplicial sets by definition; thus $d$ preserves cofibrations, weak equivalences, and colimits (it is left adjoint to a functor $d_{*}$ : cf. [4]). Its right adjoint $d_{*}$ preserves trivial fibrations, hence pointwise trivial fibrations of diagrams of simplicial sets.

The left adjoint $d^{*}$ of $d$ preserves colimits by definition, and if $c: A \hookrightarrow B$ is any trivial cofibration of simplicial sets, then $d^{*}(c)$ is a trivial Moerdijk cofibration by adjointness and CM4 for the Moerdijk closed model structure. Any weak equivalence $e: X \rightarrow Y$ of simplicial sets factors as

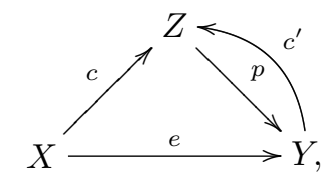

where $c$ is a trivial cofibration and $p$ is left inverse to a trivial cofibration $c^{\prime}$, thus $d^{*}$ sends weak equivalences of simplicial sets to Moerdijk weak equivalences (also, $d^{*}$ 
sends cofibrations to Moerdijk cofibrations by another adjointness plus CM4 argument). The right adjoint $d$ of $d^{*}$ preserves trivial fibrations by definition of the Moerdijk structure, so it preserves pointwise trivial fibrations of diagrams of bisimplicial sets.

The composite $d d_{*}$ therefore preserves pointwise trivial fibrations of diagrams of simplicial sets, so its left adjoint $d d^{*}$ preserves projective cofibrations. As $d$ and $d^{*}$ both preserve weak equivalences and colimits, $d d^{*}$ preserves projective cofibrant models of diagrams of simplicial sets and therefore homotopy colimits.

Observe that

$$
d d^{*}\left(\Delta^{n}\right)=d \Delta^{n, n}=\Delta^{n} \times \Delta^{n} \simeq *
$$

for $n \geqslant 0$ so that the canonical maps $\eta: \Delta^{n} \rightarrow d d^{*}\left(\Delta^{n}\right)$ are weak equivalences for $n \geqslant 0$. For any simplicial set $X$ there is a canonical weak equivalence

$$
\stackrel{\text { holim}}{\longrightarrow} \Delta / X \Delta^{n} \simeq X
$$

(cf. $[4,5.2, \mathrm{IV}])$. Consider the commutative square

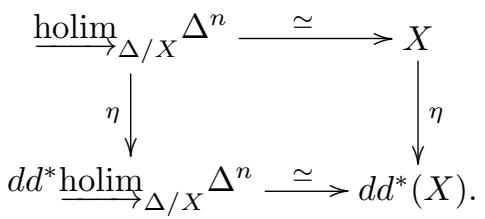

The top and bottom maps are weak equivalences, and the left-hand map is a weak equivalence as it is weakly equivalent to the map $\stackrel{\text { holim}}{\longrightarrow} \Delta / X_{X} \eta_{n}$ where the

$$
\eta_{n}: \Delta^{n} \rightarrow d d^{*}\left(\Delta^{n}\right)
$$

are the unit maps for $\left(d^{*}, d\right)$ applied to $\Delta^{n}$ for $n \geqslant 0$.

Corollary 4.10. The adjunction $\left(d^{*}, d\right)$ is a Quillen equivalence between the standard closed model structure on simplicial sets, and the Moerdijk closed model structure on bisimplicial sets.

Proof. The functor $d^{*}$ preserves cofibrations and weak equivalences by the proof of the previous lemma, and $d$ preserves fibrations and weak equivalences by definition of the Moerdijk closed model structure; hence $\left(d^{*}, d\right)$ is a Quillen adjunction. If $f: X \rightarrow d Y$ is a weak equivalence of simplicial sets, then the adjoint map $\tilde{f}: d^{*} X \rightarrow Y$ defined by the adjunction diagram

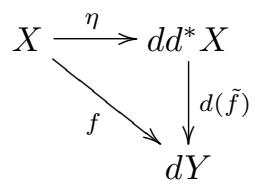

is a diagonal weak equivalence since $\eta$ is a weak equivalence. Conversely, suppose $\tilde{f}: d^{*} X \rightarrow Y$ is a diagonal weak equivalence. Then $d(\tilde{f})$ is a weak equivalence so the composite $f=d(\tilde{f}) \eta$ is a weak equivalence, as was to be shown. 
Let $i^{\prime \prime \prime}$ denote the inclusion functor

$$
i^{\prime \prime \prime}: H_{d}(x, z) \hookrightarrow H(x, z) .
$$

Again, $H_{d}(x, z)$ is not a priori a full subcategory of $H(x, z)$.

Lemma 4.11. For any two pointed simplicial sheaves $x$ and $z$, the induced map $\pi_{0}\left(i^{\prime \prime \prime}\right)$ is injective.

Proof. Suppose $m:(f, p) \rightarrow(g, q)$ is any morphism of $H(x, z)$. Then $x \times z=d(x \times z)$ so that the map $(f, p): u \rightarrow x \times z$ uniquely factors as

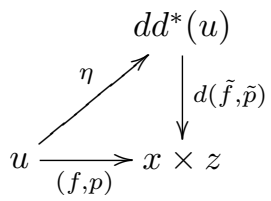

for some maps $\tilde{f}, \tilde{p}$ from $d^{*}(u)$ to $x, z$ by adjointness, and similarly $(g, q): v \rightarrow x \times z$ factors as $\eta$ followed by a uniquely determined pair $(\tilde{g}, \tilde{q})$ from $d^{*}(v)$ to $x \times z$. There is then a commutative diagram

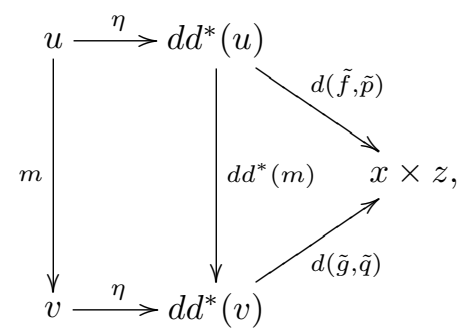

where both maps $\eta$ and the map $m$ are local weak equivalences (for $\eta$ use Lemma 4.9 in sections), so $d d^{*}(m)$ is a local weak equivalence. Further, $d(\tilde{f})$ is a local weak equivalence since $f$ and $\eta$ are local weak equivalences, so $\tilde{f}$ is a diagonal local weak, and equivalence and similarly for $\tilde{g}$. The zigzag

$$
(f, p) \stackrel{\eta}{\rightarrow}(d(\tilde{f}), d(\tilde{p})) \stackrel{d d^{*}(m)}{\longrightarrow}(d(\tilde{g}), d(\tilde{q})) \stackrel{\eta}{\leftarrow}(g, q)
$$

in $H(x, z)$ shows that the original map $m$ is in the same path component as $d d^{*}(m)$, thus any morphism $m$ of objects in $H(x, z)$ naturally lifts to a morphism $d d^{*}(m)$ in $H_{d}(x, z)$. It follows that any zigzag of maps in $H(x, z)$ naturally lifts to a zigzag of maps in $H_{d}(x, z)$, so the result follows.

Corollary 4.12. Suppose $x$ and $z$ are two pointed simplicial sheaves as above such that $z$ is locally fibrant. Then the induced maps

$$
\pi_{0}\left(i^{\prime \prime \prime}\right): \pi_{0} H_{d}(x, z) \rightarrow \pi_{0} H(x, z)
$$

and

$$
\pi_{0}\left(i^{\prime \prime}\right): \pi_{0} H_{d-\text { hyp }}(x, z) \rightarrow \pi_{0} H_{\text {hyp }}(x, z)
$$

are bijections. 
Proof. The map $\pi_{0}\left(i^{\prime \prime \prime}\right)$ is injective by the previous lemma. Consider the commutative square

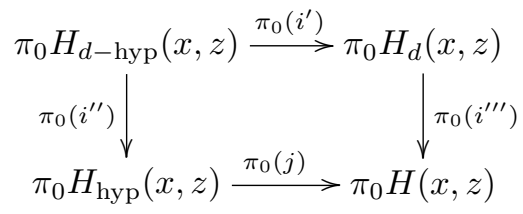

induced by the corresponding inclusions. The top and bottom maps are bijections and the left-hand vertical map is surjective, so $\pi_{0}\left(i^{\prime \prime \prime}\right)$ is surjective, hence bijective by the previous lemma. But then the composite $\pi_{0}\left(i^{\prime \prime \prime}\right) \pi_{0}\left(i^{\prime}\right)$ is bijective so $\pi_{0}\left(i^{\prime \prime}\right)$ must also be injective, hence bijective.

Recall as above that $H_{\text {bihyp }}(x, y)$ is a full subcategory of $H_{d}(x, y)$ for any fixed choice of pointed simplicial sheaves $x$ and $y$. Letting

$$
i: H_{\text {bihyp }}(x, y) \hookrightarrow H_{d}(x, y)
$$

denote the inclusion functor, one therefore knows that the induced map $\pi_{0}(i)$ on path components is injective. To move towards bijectivity one begins with an analogue of Corollary 4.2 :

Lemma 4.13. If $f: X \rightarrow Y$ is a weak equivalence of fibrant simplicial sets, then the induced map $d_{*}(f): d_{*} X \rightarrow d_{*} Y$ is a degreewise weak equivalence of bisimplicial sets.

Proof. By Brown's factorization lemma it suffices to show that $d_{*}$ sends trivial fibrations of simplicial sets to degreewise trivial fibrations of bisimplicial sets. The induced $\operatorname{map} d_{*}(f)$ is a degreewise trivial fibration if and only if it has the right lifting property with respect to all Bousfield-Kan cofibrations $c: A \rightarrow B$. By adjunction such lifting problems correspond to lifting problems

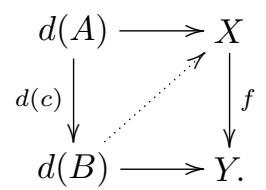

These all have solutions since $c$ is, in particular, a pointwise cofibration, hence $d(c)$ is a cofibration and the lift exists since $f$ was a trivial fibration by assumption.

Here is the local version:

Lemma 4.14. If $\beta: z \rightarrow z^{\prime}$ is a pointed local weak equivalence of pointed locally fibrant simplicial sheaves, then $d_{*}(\beta)$ is a pointed degreewise local weak equivalence of bisimplicial sheaves.

Proof. Fix a Boolean localization $p: \operatorname{Shv}(\mathcal{B}) \rightarrow \operatorname{Shv}(\mathcal{C})$. Then $p^{*}(\beta)$ is a sectionwise weak equivalence of sectionwise fibrant simplicial sheaves on $\mathcal{B}$ so $d_{*}\left(p^{*}(\beta)\right)$ is a degreewise weak equivalence in each section by Lemma 4.13. By the argument of Lemma 4.3 it follows that $p^{*}\left(d_{*}(\beta)\right)$ is also a degreewise weak equivalence in each section, or equivalently a sectionwise weak equivalence in each degree, so $d_{*}(\beta)$ is a local weak equivalence in each degree. The map $d_{*}(\beta)$ is automatically pointed so the result follows. 
Lemma 4.15. If $f: x \rightarrow y$ is a pointed local fibration of simplicial sheaves, then $d_{*}(f)$ is a pointed degreewise local fibration of bisimplicial sheaves.

Proof. Fix a Boolean localization $p: \operatorname{Shv}(\mathcal{B}) \rightarrow \operatorname{Shv}(\mathcal{C})$. Then $p^{*}(f)$ is a sectionwise fibration of simplicial sheaves on $\mathcal{B}$. An adjunction argument (starting from the fact that the diagonal of a degreewise trivial cofibration is a trivial cofibration) shows that $d_{*}\left(p^{*}(f)\right)$ is a degreewise fibration in each section, or alternatively a sectionwise fibration in each degree. Thus $p^{*}\left(d_{*}(f)\right)$ is a sectionwise fibration in each degree so $d_{*}(f)$ is a local fibration in each degree, as was to be shown.

One has the following analogue of Lemma 4.4:

Lemma 4.16. If $\beta: z \rightarrow z^{\prime}$ is a local weak equivalence of pointed locally fibrant simplicial sheaves, then the induced functor

$$
\beta_{*}: B H_{\mathrm{bihyp}}(x, z) \rightarrow B H_{\mathrm{bihyp}}\left(x, z^{\prime}\right)
$$

is a homotopy equivalence.

Proof. The proof is analogous to that of Lemma 4.4: the functor $\beta_{*}$ is defined by composition with $\beta$, and the induced map $d_{*}(\beta)$ is a degreewise local weak equivalence by Lemma 4.14. Supposing one has a "cocycle" of the form

$$
x \stackrel{f}{\leftarrow} u \stackrel{\tilde{p}}{\rightarrow} d_{*}\left(z^{\prime}\right),
$$

where $f$ is a bisimplicial hypercover, one factors the map $(f, \tilde{p})$ as a sectionwise degreewise weak equivalence $c$ followed by a sectionwise degreewise fibration $\left(p_{x}, \tilde{p}_{z^{\prime}}\right)$. The map $p_{x}$ is a degreewise local weak equivalence since $c$ and $f$ are and is a degreewise local fibration since it equals the composite

$$
w \stackrel{\left(p_{x}, \tilde{p}_{z^{\prime}}\right)}{\longrightarrow} x \times d_{*}\left(z^{\prime}\right) \stackrel{\mathrm{pr}_{L}}{\longrightarrow} x,
$$

where the former map is a sectionwise fibration in each degree and the latter map is a degreewise local fibration by Lemma 4.15 ; thus $p_{x}$ is again a bisimplicial hypercover. The map $1_{x} \times d_{*}(\beta)$ is a degreewise local weak equivalence so its pullback $\left(1_{x} \times d_{*}(\beta)\right)_{*}$ along $\left(p_{x}, \tilde{p}_{z^{\prime}}\right)$ is also a degreewise local weak equivalence by a Boolean localization argument. The other pullback map $\left(p_{x}^{*}, \tilde{p}_{z}^{*}\right)$ is a sectionwise degreewise fibration since $\left(p_{x}, \tilde{p}_{z^{\prime}}\right)$ was, so $p_{x}^{*}$ is also a degreewise local fibration. The map $p_{x}^{*}$ is also a degreewise local weak equivalence since $p_{x}, 1_{x} \times d_{*}(\beta)$, and $\left(1_{x} \times d_{*}(\beta)\right)_{*}$ are, so $p_{x}^{*}$ is also a bisimplicial hypercover. This construction determines the functor $\tilde{\psi}$, and the remainder of the argument follows the proof of Lemma 4.4 verbatim.

Lemma 4.17. Suppose $x$ and $y$ are pointed simplicial sheaves on a pointed small Grothendieck site $\mathcal{C}$ with y locally fibrant. Then the map

$$
\pi_{0}(i): \pi_{0} H_{\text {bihyp }}(x, y) \rightarrow \pi_{0} H_{d}(x, y)
$$

induced by inclusion is a bijection.

Proof. First assume that $y$ is globally fibrant. Starting with any "cocycle"

$$
x \stackrel{f}{\leftarrow} u \stackrel{\tilde{p}}{\rightarrow} d_{*}(y)
$$

corresponding to an object of $H_{d}(x, y)$, factor $f$ as a sectionwise degreewise cofibration $c$ followed by a sectionwise degreewise trivial fibration $h$. Then $h$ is a bisimplicial 
hypercover, and one has an induced map of cocycles

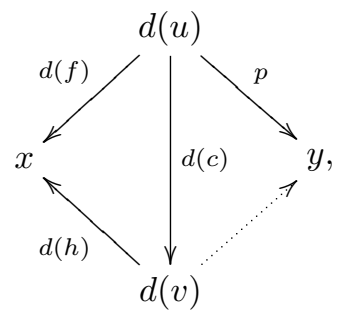

where $d(f)$ and $d(h)$ are local weak equivalences so $d(c)$ is a local weak equivalence as well as a cofibration and the lift therefore exists since $y$ was globally fibrant by assumption.

More generally, suppose $y$ is locally fibrant and fix a pointed globally fibrant replacement $\beta: y \rightarrow z$ for $y$. Then there is a commutative square

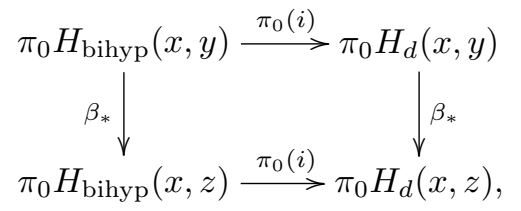

where both vertical maps $\beta_{*}$ are induced by composition with $\beta$ so they are bijections by Lemmas 4.16 and 4.4, and the bottom map is a bijection by the previous paragraph so the top map is a bijection as well, as was to be shown.

The results above may be summarized as follows:

Theorem 4.18. Suppose $x$ and $y$ are pointed simplicial sheaves on a pointed small Grothendieck site $\mathcal{C}$, where $y$ is locally fibrant. Then there are canonical bijections

$$
\pi_{0} H_{\text {bihyp }}(x, y) \cong \pi_{0} H_{d-\text { hyp }}(x, y) \cong \pi_{0} H_{d}(x, y) \cong \pi_{0} H_{\text {hyp }}(x, y) \cong \pi_{0} H(x, y) \cong[x, y] \text {. }
$$

Proof. The latter bijection is a consequence of Theorem 1 of [10]. The remaining bijections have already been established.

Corollary 4.19. With the hypotheses of Theorem 4.18, there are canonical bijections

$$
\pi_{0} H_{\text {bihyp }}(x, y) \stackrel{\cong}{\longrightarrow} \pi_{0} H_{\text {bihyp }}\left(x^{\prime}, y^{\prime}\right)
$$

induced by any two local weak equivalences $\alpha: x \rightarrow x^{\prime}, \beta: y \rightarrow y^{\prime}$ of pointed locally fibrant simplicial sheaves on $\mathcal{C}$.

Proof. Use the analogous property for $H(x, y)$, proven in Lemma 1 of [10].

\section{Applications to étale homotopy theory}

Étale homotopy types are constructed using hypercovers and simplicial homotopy classes of maps between them, so it becomes necessary to consider some cocycle categories that use these classes of maps in their definitions. 
Inspired by [11], consider the cocycle category $H_{\text {bihyp }}^{h^{\prime}}(x, y)$ whose objects are cocycles of the form

$$
x \stackrel{d(f)}{\longleftarrow} d(u) \stackrel{[p]}{\longrightarrow} y
$$

for pointed bisimplicial hypercovers $u$ of $x$ and whose morphisms are diagrams

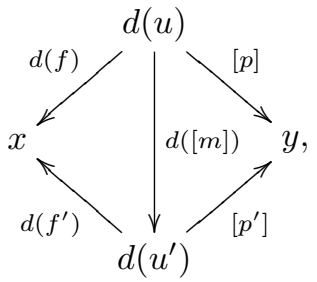

where square brackets indicate simplicial homotopy classes of maps, the middle maps are induced by fibrewise simplicial homotopy classes $[m]: u \rightarrow u^{\prime}$ of maps of pointed bisimplicial hypercovers of $x$, and $\left[p^{\prime}\right][d(m)]=[p]$ as pointed simplicial homotopy classes. The set of path components $\pi_{0} H_{\text {bihyp }}^{h^{\prime}}(x, y)$ is given by the colimit

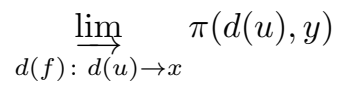

whose index category is that of pointed bisimplicial hypercovers of $x$ and pointed fibrewise simplicial homotopy classes of maps between them. The functor $\omega: H_{\text {bihyp }}(x, y)$ $\rightarrow H_{\text {bihyp }}^{h^{\prime}}(x, y)$ defined on objects by $(d(f), p) \mapsto(d(f),[p])$ is obviously surjective on path components.

Introduce another cocycle category $H_{\text {bihyp }}^{h}(x, y)$ whose objects are of the form

$$
x \stackrel{[d(f)]}{\longleftarrow} d(u) \stackrel{[p]}{\longrightarrow} y
$$

and whose morphisms are commutative diagrams

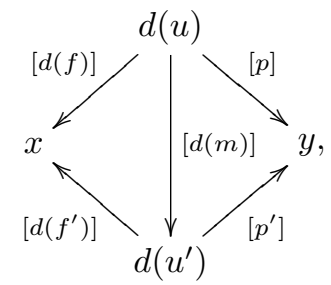

where the middle maps are induced by maps $m: u \rightarrow u^{\prime}$ of pointed bisimplicial hypercovers of $x$. One readily verifies that the maps $(d(f),[p]) \mapsto([d(f)],[p])$ on objects and $d([m]) \mapsto[d(m)]$ on morphisms determine a functor $\omega^{\prime}: H_{\text {bihyp }}^{h^{\prime}}(x, y) \rightarrow H_{\text {bihyp }}^{h}(x, y)$ which again is obviously surjective on path components.

To relate these observations back to a homotopy category, suppose that the point $x:$ Set $\rightarrow \operatorname{Shv}(\mathcal{C})$ comes from an object $\Omega$ of $\mathcal{C}$ representing a sheaf in the sense that the inverse image functor $x^{*}$ is given by a composite

$$
\operatorname{Shv}(\mathcal{C}) \stackrel{?}{\longrightarrow} \operatorname{Shv}(\mathcal{C} / \Omega) \stackrel{\Gamma_{*}}{\longrightarrow} \operatorname{Set}
$$

defined by first restricting to the site $\mathcal{C} / \Omega$ and then taking global sections. This is exactly the situation in étale homotopy theory when one works on a "big" étale site 
containing the separably closed field $\Omega:=\operatorname{Spec} \Omega$ which is used to give the geometric point $x$ of the base scheme or DM stack $S$. A pointed (bi)simplicial sheaf $(X, z)$ on such a site $(\mathcal{C}, x)$ then corresponds exactly to a section

$$
\Omega \stackrel{z}{\rightarrow} X,
$$

where $\Omega=\Omega \stackrel{x}{\rightarrow} S$ is the object of $\mathcal{C}$ corresponding to the point $x$, and a pointed map $(X, z) \rightarrow\left(Y, z^{\prime}\right)$ corresponds exactly to a map $X \rightarrow Y$ respecting the sections $z$ and $z^{\prime}$. By general nonsense there is a closed model structure on the category $\Omega / \operatorname{sShv}(\mathcal{C})$ of pointed simplicial sheaves, where the fibrations (resp. cofibrations, resp. weak equivalences) are those maps $(X, z) \rightarrow\left(Y, z^{\prime}\right)$ under $\Omega$ such that the underlying maps $X \rightarrow Y$ are fibrations (resp. cofibrations, resp. weak equivalences). Lemma 1 of $[\mathbf{1 1}]$ then says that the canonical map

$$
\pi_{0} H(x, y) \rightarrow[x, y]
$$

defined by sending any pointed cocycle $(f, g)$ to the composite $g f^{-1}$ is a bijection, where $[x, y]$ denotes morphisms in $\operatorname{Ho}(\Omega / \operatorname{sShv}(\mathcal{C}))$.

Lemma 5.1. Suppose $(\mathcal{C}, x)$ is a pointed small Grothendieck site such that the point $x$ : Set $\rightarrow \operatorname{Shv}(\mathcal{C})$ is determined by an object $\Omega$ of $\mathcal{C}$ as above which represents a sheaf (or suppose $\Omega=\emptyset$ ). Then for any two pointed simplicial sheaves $x$ and $y$ on $\mathcal{C}$ with y locally fibrant there are canonical bijections

$$
\pi_{0} H_{\text {bihyp }}(x, y) \stackrel{\pi_{0}(\omega)}{\longrightarrow} \pi_{0} H_{\text {bihyp }}^{h^{\prime}}(x, y) \stackrel{\pi_{0}\left(\omega^{\prime}\right)}{\longrightarrow} \pi_{0} H_{\text {bihyp }}^{h}(x, y) \stackrel{p}{\rightarrow}[x, y] .
$$

Proof. The displayed composite $c=p \pi_{0}\left(\omega^{\prime}\right) \pi_{0}(\omega)$ factors as

$$
\pi_{0} H_{\text {bihyp }}(x, y) \stackrel{\pi_{0}\left(j^{\prime}\right)}{\longrightarrow} \pi_{0} H(x, y) \stackrel{\cong}{\rightrightarrows}[x, y],
$$

where $j^{\prime}: H_{\text {bihyp }}(x, y) \rightarrow H(x, y)$ is the inclusion functor and the second arrow is the canonical map sending any cocycle $(f, g)$ to the composite $g f^{-1}$ in the homotopy category. As $j^{\prime}=i^{\prime \prime \prime} i, \pi_{0}\left(j^{\prime}\right)$ is a bijection by Corollary 4.12 and Lemma 4.17 so that $c$ is also a bijection. This implies that the canonical map $p$ is a surjection and that $\pi_{0}(\omega)$ is an injection, hence a bijection. But then $p \pi_{0}\left(\omega^{\prime}\right)$ is also a bijection, so $\pi_{0}\left(\omega^{\prime}\right)$ is an injection, hence a bijection, and thus $p$ is also a bijection, as was to be shown.

Here is an immediate application:

Proposition 5.2. Suppose $\mathcal{C}$ is a small Grothendieck site, $H$ a sheaf of groups on $\mathcal{C}$, $n \geqslant 0$ if $H$ is abelian or $0 \leqslant n \leqslant 1$ otherwise, and $X$ a simplicial sheaf on $\mathcal{C}$. Then there are canonical bijections

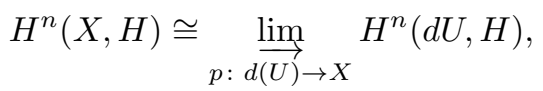

where the colimit is indexed over the category of bisimplicial hypercovers of $X$ and fibrewise simplicial homotopy classes of maps between them. If $X$ is representable, then these extend to bijections

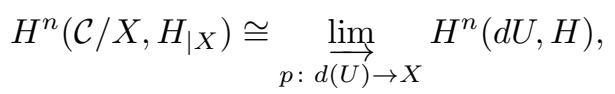

where $H_{\mid X}$ is defined by $H_{\mid ?}\left(U \rightarrow X_{n}\right):=H(U)$. 
Proof. There is a series of identifications

$$
\begin{aligned}
& H^{n}(X, H):=[X, K(H, n)] \\
& \cong \lim _{p: d(U) \rightarrow X} \pi(d U, G K(H, n)) \\
& \cong \lim _{p: d(U) \rightarrow X}[d U, G K(H, n)] \\
& =\lim _{p: d(U) \rightarrow X} H^{n}(d U, H),
\end{aligned}
$$

where $G K(H, n)$ is a globally fibrant model for $K(H, n)$, the first isomorphism is by Lemma 5.1 and the identification of $\pi_{0} H_{\text {bihyp }}^{h^{\prime}}(X, G K(H, n))$, and the final identification is, by definition, of cohomology of $d U$ with coefficients in $H$. The latter statement follows from the proof of Theorem 3.10 of [7].

One may compare this with the statement $([\mathbf{3}, 3.8])$, keeping in mind that the bisimplicial hypercovers $U \rightarrow X$ here are not assumed to be representable. This argument works even for nonabelian cohomology $([\mathbf{3}, 3.8]$ only addressed the abelian case).

\section{1. Étale homotopy types from bisimplicial hypercovers}

To return to the étale homotopy type $T(X, z)$ defined in Section 2, one is motivated by the above results to consider the diagram $T_{b}(X, z)$ for any pointed connected simplicial sheaf $x=(X, z)$ on a pointed locally connected small Grothendieck site $\mathcal{C}$ given by the simplicial sets $\Pi d(u)$ for pointed bisimplicial hypercovers $u \rightarrow x$, with maps induced by the pointed fibrewise simplicial homotopy classes of maps between the bisimplicial hypercovers over $x$. That this determines a pro-object in Ho(sSet $\left.{ }_{*}\right)$ is a consequence of the functoriality of the construction of equalizers for simplicial homotopy classes of maps after noting that the sheaves $X_{m}$ are globally, hence locally, fibrant.

The following result, which is the main point of this work, gives the relationship between $T(X, z)$ and $T_{b}(X, z)$ for locally fibrant $X$ :

Theorem 5.3. Suppose $\mathcal{C}$ is a pointed locally connected small Grothendieck site such that the point $\mathbf{S e t} \rightarrow \operatorname{Shv}(\mathcal{C})$ is determined by some object $\Omega$ representing a sheaf, and $x=(X, z)$ a pointed connected locally fibrant simplicial sheaf on $\mathcal{C}$. Then the proobject $T(X, z)$ of $\mathrm{Ho}\left(\mathbf{s S e t}_{*}\right)$ is canonically pro-isomorphic to the pro-object $T_{b}(X, z)$, and similarly for the unpointed variants in $\mathrm{Ho}(\mathbf{s S e t})$. Furthermore, these pro-isomorphisms $T(X, z) \cong T_{b}(X, z)$ are functorial in $(X, z)$, and similarly for the unpointed case.

Proof. For the first part it suffices to give a canonical natural isomorphism between the functors that these pro-objects pro-represent. On the one hand one has canonical 
isomorphisms

$$
\begin{aligned}
{\left[T_{b}(X, z), y\right]: } & {\underset{\lim }{p: \overrightarrow{d(u)} \rightarrow x}}[\Pi d(u), y] \\
& \cong{\underset{\lim }{d(u) \rightarrow x}} \pi(\Pi d(u), y) \\
& \cong \lim _{p: \overrightarrow{d(u)} \rightarrow x} \pi\left(d(u), \Gamma^{*} y\right) \\
& \cong \pi_{0} H\left(x, \Gamma^{*} y\right) \\
& \cong\left[x, \Gamma^{*} y\right]
\end{aligned}
$$

natural in $y$ by Theorem 4.18 and Lemma 5.1, where one may assume $y$ is fibrant by applying the $\mathrm{Ex}^{\infty}$ functor. By a similar calculation one has $[T(X, z), y] \cong\left[x, \Gamma^{*} y\right]$ naturally in $y$. Therefore there are canonical natural bijections $\left[T_{b}(X, z), y\right] \cong[T(X, z), y]$, so the functors $\left[T_{b}(X, z),-\right]$ and $[T(X, z),-]$ are canonically naturally isomorphic and thus the representing pro-objects are canonically pro-isomorphic, as was to be shown. Forgetting the points yields the analogous statement for the unpointed case.

For functoriality, observe that the cocycle categories

$$
H_{\text {bihyp }}\left(x, \Gamma^{*} y\right) \text { and } H_{\text {hyp }}\left(x, \Gamma^{*} y\right)
$$

determine contravariant functors $f^{*}$ in $x$ by sending any cocycle of the form

$$
x \stackrel{d(p)}{\longleftarrow} d(u) \stackrel{q}{\rightarrow} \Gamma^{*} y
$$

to the cocycle $\left(d\left(p_{*}\right), q \cdot d\left(\operatorname{pr}_{u}\right)\right): d\left(u^{\prime}\right) \rightarrow x^{\prime} \times \Gamma^{*} y$ induced by pulling back $p$ to $p_{*}$ along any pointed map $f: x^{\prime} \rightarrow x$ of pointed simplicial sheaves, and similarly for $H_{\text {hyp }}$. Consider the diagram

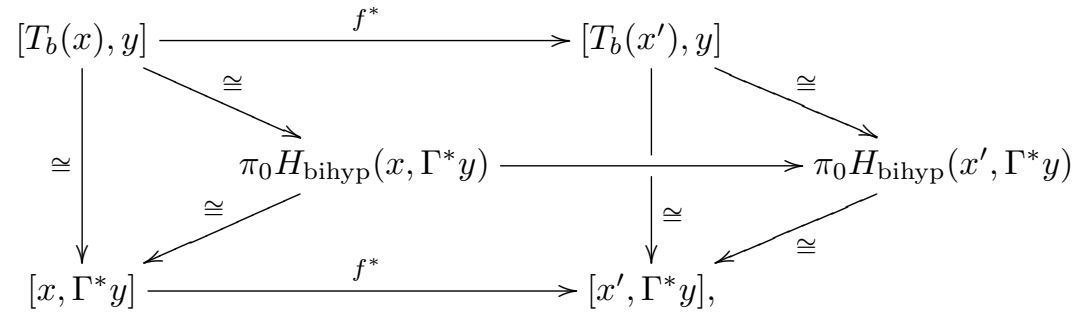

where the two triangles on either end are canonical factorizations of the canonical natural isomorphisms above. The back square commutes if the top and the front squares commute. Any element of the set

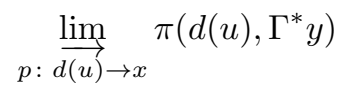

is represented by some pair $\left(p: u \rightarrow x,[q]: d(u) \rightarrow \Gamma^{*} y\right)$, where $p$ is a pointed bisimplicial hypercover, and $f^{*}$ (precomposition with $f$ ) on $\left[T_{b}(x), y\right]$ induces a map $f^{*}$ sending such a pair to the element of

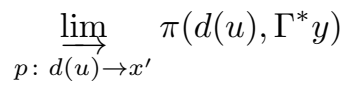

represented by the pair $\left(p_{*}: u^{\prime} \rightarrow x^{\prime},\left[q \cdot d\left(\operatorname{pr}_{u}\right)\right]\right)$; clearly this is compatible with the 
functor $\pi_{0} H_{\text {bihyp }}\left(-, \Gamma^{*} y\right)$ so the top square commutes. Chasing the cocycle $(d(p), q)$ around the front square gives

$$
(d(p), q) \mapsto\left[q d(p)^{-1}\right] \mapsto\left[q d(p)^{-1} f\right]
$$

on the left side and

$$
(d(p), q) \mapsto\left(d\left(p_{*}\right), q \cdot d\left(\mathrm{pr}_{u}\right)\right) \mapsto\left[q d\left(\mathrm{pr}_{u}\right) d\left(p_{*}\right)^{-1}\right]
$$

on the other, but these are equal since $d(p) d\left(\mathrm{pr}_{u}\right)=f d\left(p_{*}\right)$ by definition of $p_{*}$. Thus the front square also commutes, so the back square commutes. A similar computation with $\pi_{0} H_{\text {hyp }}\left(-, \Gamma^{*} y\right)$ shows that the analogous square commutes, giving the functoriality in $x$. The unpointed case follows by the same argument.

To move towards a statement about the invariance of $T_{b}(X, z)$ under local weak equivalences, there is

Lemma 5.4. Under the same assumptions on $\mathcal{C}$ as in Theorem 5.3, the pro-object $T_{b}(X, z)$ associated to any pointed connected simplicial sheaf $x=(X, z)$ on $\mathcal{C}$ is canonically pro-isomorphic to the pro-object $T_{b}\left(\mathrm{Ex}^{\infty}(X), z^{\prime}\right)$ in $\mathrm{Ho}\left(\mathbf{s S e t}_{*}\right)$ where $z^{\prime}$ is induced by the canonical sectionwise weak equivalence $\eta_{X}: X \rightarrow \mathrm{Ex}^{\infty}(X)$, and similarly for the unpointed variants in $\mathrm{Ho}(\mathbf{s S e t})$. Furthermore, these pro-isomorphisms $T_{b}(X, z) \cong T_{b}\left(\mathrm{Ex}^{\infty}(X), z^{\prime}\right)$ are functorial in $(X, z)$, and similarly for the unpointed case.

Proof. For the first statement, observe that there are canonical bijections

$$
\begin{aligned}
{\left[T_{b}\left(\operatorname{Ex}^{\infty}(X), z^{\prime}\right), y\right] } & :=\underset{p: d(u) \rightarrow \lim _{\rightarrow \infty}(X)}{ }[\Pi d(u), y] \\
& \cong \pi_{0} H_{\text {bihyp }}^{h^{\prime}}\left(\operatorname{Ex}^{\infty}\left(X, z^{\prime}\right), \Gamma^{*} y\right) \\
& \cong \pi_{0} H\left(\operatorname{Ex}^{\infty}\left(X, z^{\prime}\right), \Gamma^{*} y\right) \\
& \cong\left[\operatorname{Ex}^{\infty}\left(X, z^{\prime}\right), \Gamma^{*} y\right] \\
& \cong\left[x, \Gamma^{*} y\right] \\
& \cong\left[T_{b}(X, z), y\right]
\end{aligned}
$$

natural in $y$ for any fibrant simplicial set $y$ by Lemma 5.1, Theorem 4.18, Lemma 1 of [10], and Theorem 1 of [10]. These naturally extend to canonical bijections for arbitrary $y$ via $\mathrm{Ex}^{\infty}$. After the proof of Theorem 5.3, establishing functoriality in $x$ reduces to verifying that the square

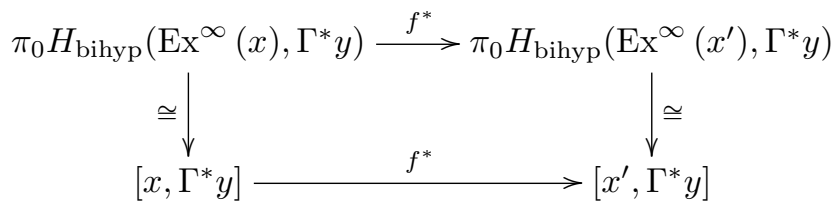

commutes. Chasing a cocycle $(d(p), q): d(u) \rightarrow \operatorname{Ex}^{\infty}(x) \times \Gamma^{*} y$ around this square 
gives

$$
(d(p), q) \mapsto\left[q d(p)^{-1} \eta_{x}\right] \mapsto\left[q d(p)^{-1} \eta_{x} f\right]
$$

on the left side and

$$
(d(p), q) \mapsto\left(d\left(p_{*}\right), q \cdot d\left(\mathrm{pr}_{u}\right)\right) \mapsto\left[q \cdot d\left(\mathrm{pr}_{u}\right) d\left(p_{*}\right)^{-1} \eta_{x^{\prime}}\right]
$$

on the right side, but these are equal by the definition of $p_{*}$ and the fact that $\mathrm{Ex}^{\infty}$ is a functor.

The key point here is that $T_{b}(X, z)$ exists without the requirement that $X$ be locally fibrant, and the cocycle category techniques of [10] make no fibrancy assumptions on $X$. Here is the major consequence:

Corollary 5.5. With the same hypotheses as Theorem 5.3 on $\mathcal{C}$, suppose $x=(X, z)$ and $y=(Y, y)$ are pointed connected simplicial sheaves on $\mathcal{C}$, and $f:(Y, y) \rightarrow(X, z)$ a pointed local weak equivalence. Then the strict morphism of pro-objects $f_{*}: T_{b}(Y, y) \rightarrow$ $T_{b}(X, z)$ induced from $f$ by pulling back bisimplicial hypercovers along $f$ is a proisomorphism, and similarly for the unpointed setting.

Proof. The pointed map $f$ induces a pointed map $\eta_{f}: \operatorname{Ex}^{\infty}(Y) \rightarrow \operatorname{Ex}^{\infty}(X)$ by functoriality of $\mathrm{Ex}^{\infty}$. As $\operatorname{Ex}^{\infty}(X)$ and $\operatorname{Ex}^{\infty}(Y)$ are locally fibrant and connected there are canonical natural isomorphisms

$$
\left[T_{b}\left(\operatorname{Ex}^{\infty}(X)\right),-\right] \cong\left[T\left(\operatorname{Ex}^{\infty}(X)\right),-\right]
$$

and similarly for $\operatorname{Ex}^{\infty}(Y)$ by Theorem 5.3. Then there is a commutative diagram

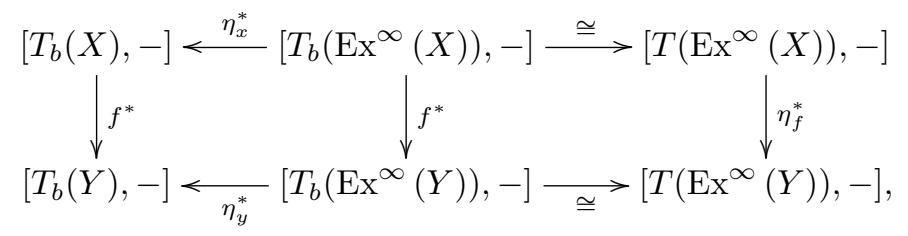

where $\eta_{f}^{*}$ is a natural isomorphism by Proposition 3.3. The right-hand square is commutative by the functoriality in Theorem 5.3 so the middle $f^{*}$ is also a natural isomorphism. The left-hand square commutes by the functoriality in Lemma 5.4, and the maps $\eta_{x}^{*}$ and $\eta_{y}^{*}$ are natural isomorphisms since $\eta_{x}$ and $\eta_{y}$ are local weak equivalences, so the map $f^{*}$ on the left is a natural isomorphism. This map $f^{*}$ was induced by precomposition with the map $f_{*}: T_{b}(Y, y) \rightarrow T_{b}(X, z)$ induced by $f$ itself, so $f_{*}$ is a pro-isomorphism, as was to be shown.

\section{References}

[1] M. Artin and B. Mazur, Etale homotopy, Lecture Notes in Math. 100, SpringerVerlag, New York, 1969.

[2] K.S. Brown, Abstract homotopy theory and generalized sheaf cohomology, Trans. Amer. Math. Soc. 186 (1973), 419-458.

[3] E.M. Friedlander, Étale homotopy of simplicial schemes, Annals of Math. Studies 104, Princeton University Press, Princeton, N.J., 1982. 
[4] P.G. Goerss and J.F. Jardine, Simplicial homotopy theory, Progress in Math. 174, Birkhäuser Verlag, Basel, 1999.

[5] D.C. Isaksen, Etale realization on the $\mathbb{A}^{1}$-homotopy theory of schemes, $A d v$. Math. 184 (2004), no. 1, 37-63.

[6] J.F. Jardine, Simplicial objects in a Grothendieck topos, in Applications of algebraic K-theory to algebraic geometry and number theory, Part I, II (Boulder, Colo., 1983), Contemp. Math. 55, pp. 193-239. Amer. Math. Soc., Providence, RI, 1986.

[7] J.F. Jardine, Simplicial presheaves, J. Pure Appl. Algebra 47 (1987), no. 1, $35-87$.

[8] J.F. Jardine, Higher spinor classes, Mem. Amer. Math. Soc. 110, no. 528, $1994, \mathrm{vi}+88$.

[9] J.F. Jardine, Boolean localization, in practice, Doc. Math. 1 (1996), no. 13, 245-275 (electronic).

[10] J.F. Jardine, Cocycle categories, in Algebraic topology, Abel Symposia 4 (2009), 185-218, Springer, Berlin.

[11] J.F. Jardine, The Verdier hypercovering theorem, Canad. Math. Bull. 55 (2012), no. 2, 319-328.

[12] A. Schmidt, On the étale homotopy type of Voevodsky's spaces, K-theory preprint archives, http://www.math.uiuc.edu/K-theory/0675, 2003.

Michael D. Misamore m.misamore@gmail.com

920 N. Calvert St., Baltimore, MD 21202, USA 